\section{CPS-010 SAFETY AND EFFICACY OF LOW MOLECULAR WEIGHT HEPARINS IN PATIENTS WITH PORTAL VEIN THROMBOSIS}

${ }^{1} \mathrm{M}$ Scaldaferri ${ }^{*},{ }^{2} \mathrm{E}$ Rolle, ${ }^{2} \mathrm{C}$ Zurlo, ${ }^{1} \mathrm{E}$ Castellana, ${ }^{2} \mathrm{GM}$ Saracco, ${ }^{1} \mathrm{~F}$ Cattel. ${ }^{1} \mathrm{C}$ itta' Della Salute E Della Scienza Di Torino, Pharmacy, Turin, Italy; ${ }^{2}$ Citta' Della Salute E Della Scienza Di Torino, Gastroenterology, Turin, Italy

\subsection{6/ejhpharm-2020-eahpconf.111}

Background and importance Portal vein thrombosis (PVT) represents a well known complication during the natural course of liver cirrhosis, ranging from asymptomatic cases to life threatening conditions related to portal hypertension and hepatic decompensation. Treatment of PVT in patients with liver cirrhosis is not well established.

Aim and objectives To assess the safety and efficacy of low molecular weight heparins (LMWH) to treat PVT in cirrhotic patients.

Material and methods Clinical charts of all patients treated with LMWH for PVT were reviewed for data on age, sex, aetiology of liver cirrhosis, presence of portal hypertension, congestive gastropathy (GC), hepatocarcinoma (HCC), treatment with LMWH, adverse events and follow-up.

Results Sixty-one patients diagnosed with PVT and cirrhosis from January 2017 to June 2019 were evaluated for anticoagulation therapy. Forty-seven patients were men, median age 61 years (range 21-84). Aetiology of cirrhosis was: alcoholic $(n=10 ; 16 \%)$, hepatitis C-HCV $(n=9 ; 14 \%)$, alcoholic +HCV $(n=7 ; 11 \%)$, hepatitis $B(n=3 ; 5 \%)$, non-alcoholic fatty liver disease/non-alcoholic steatohepatitis $(n=4$; $6 \%)$ and other/combined aetiology $(n=28 ; 48 \%)$. Portal hypertension and GC were present in 57 (93\%) and 52 $(85 \%)$ patients, respectively. Twenty-seven patients had HCC. Fifty-five patients (90\%) were diagnosed with PVT, while 1 patient had PTV and cavernoma and 3 patients had other diagnosis. Treatment was performed with nadroparin $(\mathrm{n}=24 ; 39 \%)$, enoxaparin $(\mathrm{n}=35 ; 58 \%)$ and parnaparin $(\mathrm{n}=2 ; 3 \%)$, according to hospital availability. At follow-up in June 2019, 42 patients had discontinued therapy. Reasons for discontinuation were: complete or partial recanalisation $(n=19 ; 31 \%)$, orthotopic liver transplantation $(n=10 ; 16 \%)$, death $(n=2 ; 3 \%)$, progression of liver disease $(n=3 ; 5 \%)$ and other $(\mathrm{n}=8 ; 13 \%)$. Fifty-one patients had no adverse events; the only adverse events detected were bleeding $(n=6)$ and thrombocytopenia $(n=1)$. Twenty-four patients had dose changes.

Conclusion and relevance LMWH were shown to be safe and well tolerated in our patients with only minor and transient side effects.

\section{REFERENCES AND/OR ACKNOWLEDGEMENTS}

1. Amitrano $L$, et al. Safety and efficacy of anticoagulation therapy with low molecular weight heparin for portal vein thrombosis in patients with liver cirrhosis. J Clin Gastroenterol 2010:44:448-451.

No conflict of interest.

\section{CPS-011 PRESCRIPTION ANALYSIS OF DIRECT ACTING ORAL ANTICOAGULANTS}

I Soto*, B Rubio, M Mañes, J Solis, B Bertran De Lis, EP Gómez, E Maroto, MI Bernias, I Sollano, I Morona, C Moriel. Hospital Universitario De Móstoles, Hospital Pharmacy, Móstoles, Spain

10.1136/ejhpharm-2020-eahpconf.112
Background and importance The use of direct acting oral anticoagulants (DOACs) has increased in recent years. Their posology and adverse effects require pharmaceutical monitoring in order to guarantee effective and safe treatment.

Aim and objectives To analyse the prescription and utilisation criteria for DOACs in clinical practice as well as the acceptance of the pharmaceutical recommendations made.

Material and methods This was a longitudinal prospective descriptive study of patients treated with DOACs (apixaban, rivaroxaban and dabigatran) admitted to a second level hospital (September 2018-March 2019). The information sources used were: Farmatools, Selene, Prescription Single Module and Horus. The variables analysed were: demographics, drugs prescribed, prescriptor service, indications, previous treatments, cause of the change, funding and pharmaceutical interventions. Results Seventy-three patients were analysed $(50.68 \%$ women; median age 82 years (IQR 73-87): 50.68\% were treated with apixaban, $30.14 \%$ with dabigatran and $19.18 \%$ with rivaroxaban. The main prescriber services were: cardiology (49.32\%), internal medicine (30.13\%) and geriatrics (9.59\%). Reasons for treatment were: $97.26 \%$ for atrial fibrillation, $1.37 \%$ for deep vein thrombosis and $1.37 \%$ for pulmonary thromboembolism. A total of $80.82 \%$ had previously received treatment with acenocoumarol, 5.48\% with DOACs and 13.70\% had not received previous treatment. The main reasons for the change from acenocoumarol to DOACs were: poor control of the international normalised ratio (INR) (59.32\%), vascular accident (15.26\%) and haemorrhagic event (10.7\%). Modifications from rivaroxaban to apixaban were observed in three patients: chronic kidney failure, age adjustment with kidney failure and haematological data altered. In addition, we observed a change from dabigatran to apixaban for gastritis. A total of $90.41 \%$ of patients had their treatment funded by the national health system. Dose adjustment was needed in $52.05 \%$ of patients, of which $86.84 \%$ were correctly made by the physician and $13.16 \%$ required pharmaceutical intervention due to kidney failure, age and/or weight, with a $60 \%$ acceptance rate.

Conclusion and relevance The most used DOAC was apixaban, prescribed mainly by cardiologists to patients with atrial fibrillation, as opposed to acenocoumarol, mainly prescribed by haematologists. Most patients had previously been treated with acenocoumarol, failing on this treatment due to poor INR control. Most had their treatment funded by meeting the funding criteria. Dose adjustments were carried out, receiving a highly acceptance rate.

\section{REFERENCES AND/OR ACKNOWLEDGEMENTS}

No conflict of interest.

\section{CPS-012 AUDIT OF ORAL ANTICOAGULANT PRESCRIBING; WHAT HAS CHANGED IN 4 YEARS?}

J Tuthill*, M Kieran, P Ging, C Meegan. Mater Misericordiae University Hospital, Pharmacy Department, Dublin, Ireland

10.1136/ejhpharm-2020-eahpconf.113

Background and importance The Mater Misericordiae University Hospital (MMUH) formulary recommendations for oral anticoagulants (OACs) are in line with the Health Service Executive (HSE) Medicines Management Programme. ${ }^{1}{ }^{2}$ Warfarin is the OAC of choice. Apixaban is the preferred direct oral anticoagulant (DOAC) if warfarin is unsuitable. Edoxaban, 
dabigatran and rivaroxaban are third-line options. ${ }^{1} 2$ In 2014 , warfarin was prescribed in $81 \%$ of cases in the MMUH. National data indicate DOACs are now prescribed more often than warfarin. ${ }^{2}$

Aim and objectives To identify current MMUH OAC prescribing practice and compare results with 2014 data.

Material and methods A point prevalence audit was completed in November 2018 by clinical pharmacists, across 30 wards on all patients receiving OACs. The OAC, indication, dose, prescribing team speciality and if treatment was commenced on this MMUH admission were recorded. Results were collated, analysed and compared with an identical 2014 audit.

Results More MMUH patients were prescribed OACs in 2018 $(\mathrm{n}=87)$ than in $2014(\mathrm{n}=53)(\mathrm{p}<0.01)$. Apixaban was the most commonly prescribed OAC $(48 \%)$, followed by rivaroxaban (20\%), warfarin (16\%), dabigatran (14\%) and edoxaban $(2 \%)$. In 2014, warfarin was the most commonly prescribed OAC (81\%), followed by rivaroxaban (15\%), apixaban $(2 \%)$ and dabigatran (2\%). DOAC prescribing was mainly for licensed indications and doses.

Medicines for the elderly speciality had the most patients on OACs in both $2018(n=29)$ and $2014(n=14)$. The majority of patients prescribed OACs in both 2014 and 2018 were aged 60 years or over. In 2014, all patients $<60$ years of age requiring oral anticoagulation were on warfarin. In 2018, all these patients were on DOACs. The number of patients starting OACs during MMUH admission was almost 10\% higher in $2018(\mathrm{n}=27)$ than in $2014(\mathrm{n}=11)(\mathrm{p}=0.18)$.

Conclusion and relevance Apixaban was the most commonly prescribed OAC in the MMUH. Use of warfarin has decreased from $81 \%$ in 2014 to $16 \%$ in 2018 and is now surpassed by DOAC prescribing $(\mathrm{p}<0.01)$. Increased OAC prescribing means increased pharmacy workload in terms of medication review and patient education.

\section{REFERENCES AND/OR ACKNOWLEDGEMENTS}

1. MMUH. Prescriber's guide, chapter 5.8. Information on DOACs. Last updated December 2018

2. HSE. Oral anticoagulants for stroke prevention in non-valvular atrial fibrillation. V.1.1. Medicines Management Programme. Updated September 2018.

No conflict of interest.

\section{CPS-013 EFFECT OF 2 MG INTRAVENOUS PHYTONADIONE TREATMENT ON INTERNATIONAL NORMALISED RATIO IN THE HOSPITALISED ADULT}

N Ramon*, M Rosado Ancin, A Santaolalla Sanchez, C Valdazo Martin, A Larrabeiti Echevarria, JJ Garcia Albás, R Hernanz Chaves, C Martinez. Hua, Service of Pharmacy, Vitoria-Gasteiz, Spain

\subsection{6/ejhpharm-2020-eahpconf.114}

Background and importance Phytonadione is widely used in patients with an elevated international normalised ratio (INR) in whom the goal is rapid reversal of INR to a safe range, whether in preparation for an invasive procedure or in supratherapeutic INR due to vitamin $\mathrm{K}$ antagonist (VKA) treatment. Vitamin $\mathrm{K}$ promotes liver synthesis of clotting factors (II, VII, IX, X) by an unknown mechanism; nonetheless, it has not been clearly demonstrated that phytonadione lowers the risk of major haemorrhage. Moreover, intravenous phytonadione administration is not free of side effects such as anaphylactoid reaction, overcorrection of INR or resistance to VKAs. Lack of compliance between published guidelines is probably because of the limited data available.
Aim and objectives The aim of the study was to analyse the reversal effect of INR caused to $2 \mathrm{mg}$ intravenous phytonadione treatment depending on the initial INR and to evaluate if lowering INR is directly related to the number of doses administered.

Material and methods A retrospective observational study was carried out based on data obtained from the hospital database that included all hospitalised adults treated with $2 \mathrm{mg}$ intravenous phytonadione in 2019. The analysis was developed by Stata/IC-V.15 and commandos cir means, cir, ttest and twoway scatter. The collected parameters were date of birth, sex, frequency, number of doses administered, INR values, date and hour INR values were collected and vitamin $\mathrm{K}$ administrations.

Results The study included 47 adults: 24 (51.1\%) men and 23 (48.9\%) women. Four frequencies were registered: unique dose (29.8\%), 24 hours (29.8\%), 12 hours (12.8\%) and 8 hours $(27.7 \%)$. Average age was 74.3 years $(95 \%$ CI 70.4 to 78.3). No mean difference in age was found between men and women $(p=0.32)$. There were significant differences between those adults not anticoagulated and those anticoagulated $(p=0.001 ; 12.1(95 \%$ CI 5.1 to 19.2$))$ and between VKA treated and not treated $(\mathrm{p}=0.0001 ; 14.4$ (95\% CI 7.4 to 21.4)). Pearson correlation of INR reversal was significantly related to the original INR value $(r=-0.99 \quad(95 \% \quad \mathrm{CI}$ -0.99476 to -0.98301$) ; \mathrm{p}=0.000)$ and VKAs/no VKAs $(\mathrm{r}=-0.52(95 \% \mathrm{CI}-0.70$ to -0.27$) ; \mathrm{p}=0.000)$ but was not related to the number of doses administered $(0.14 \quad(95 \% \mathrm{CI}$ -0.18 to 0.42$) ; \mathrm{p}=0.39)$ or age $(\mathrm{p}=0.12(95 \% \mathrm{CI}-0.39$ to $0.18) ; \mathrm{p}=0.44)$.

Conclusion and relevance Even though the INR is not universally accepted as a parameter for evaluating haemorrhage risk, it demonstrates that phytonadione reversal of an elevated INR depends on the VKA treatment status of the patient and the initial INR value but not the number of doses administered or age.

\section{REFERENCES AND/OR ACKNOWLEDGEMENTS}

No conflict of interest.

\section{CPS-014 CLINICAL AND ASSISTED IMPACT OF ISCHAEMIC ICTUS IN PATIENTS TREATED WITH ORAL ANTICOAGULANTS}

1J Ruiz*, 'A Juanes Borrego, 'L López Vinardell, 'A Plaza Diaz, M Puig Campmany, MA Mangues Bafalluy. 'Hospital Santa Creu I Sant Pau, Pharmacy, Barcelona, Spain; ${ }^{2}$ Hospital Santa Creu I Sant Pau, Emergency Department, Barcelona, Spain

\subsection{6/ejhpharm-2020-eahpconf.115}

Background and importance Anticoagulants are one of the therapeutic groups most frequently involved in drug related problems in the emergency services. However, the therapeutic management and the impact of assistance for those anticoagulated patients who suffer a stroke episode are not known.

Aim and objectives To describe the therapeutic management and healthcare impact of patients with atrial fibrillation treated with oral anticoagulants (OAT) admitted in an emergency services due to a thromboembolic stroke.

Material and methods This was a retrospective observational study. Adult patients ( $>18$ years old) with atrial fibrillation receiving treatment with OAT admitted for cardioembolic stroke were included (January 2017-June 2019). Anticoagulant dosing prior to the stroke episode was evaluated. The 